\title{
Mechanisms of cardiac resynchronization therapy: Inching closer to the truth
}

\author{
Prem Soman, MD, PhD, ${ }^{a}$ and Saara Sillanmäki, $M D^{b}$ \\ a Division of Cardiology, University of Pittsburgh, Pittsburgh, PA \\ b Department of Nuclear Medicine and Clinical Physiology, Kuopio University Hospital, Kuopio, \\ Finland
}

Received Jun 10, 2019; accepted Jun 10, 2019

doi: $10.1007 / \mathrm{s} 12350-019-01791-\mathrm{z}$

\section{See related article, pp. 672-684}

Cardiac Resynchronization Therapy (CRT) with multi-site pacing is a class IA indication for patients with symptomatic, severe left ventricular (LV) systolic dysfunction (ejection fraction $<35 \%$ ) with left bundle branch block (LBBB) and QRS duration $>150 \mathrm{~ms}^{1}{ }^{1} \mathrm{~A}$ lower class of recommendation is given to $\mathrm{QRS}$ durations between 120 and $150 \mathrm{~ms}$ and for non-LBBB QRS morphologies. These guideline recommendations for CRT have evolved over time. The original criteria for patient selection specified QRS duration $\geq 120 \mathrm{~ms}$ and did not include LBBB morphology. ${ }^{2}$ Clinical trials of patients who received CRT based on these original criteria reported that up to $30 \%$ of patients failed to derive a benefit in symptoms or LV systolic function. Recent data report a higher response rate in patients selected based on contemporary criteria. ${ }^{3}$

In this issue of the Journal, Zhang et al. analyze retrospective data to determine whether directing the $\mathrm{LV}$ lead to a viable myocardial segment that is activated late results in improved response to CRT. ${ }^{4}$ The hypothesis for this analysis stems from presumed CRT mechanisms that as yet remain poorly explained. Small, single-center studies have contributed pieces to the larger puzzle of how CRT mediates its benefit on symptoms, LV function, and prognosis. For example, studies have shown that a large scar burden in the LV precludes improvement of LV function after CRT. ${ }^{5}$ Similarly, prior

\footnotetext{
Reprint requests: Prem Soman, MD, PhD, Division of Cardiology, University of Pittsburgh, A-429 Scaife Hall, 200 Lothrop Street, Pittsburgh, PA 15213, USA; premsoman@usa.net J Nucl Cardiol 2021;28:685-7. $1071-3581 / \$ 34.00$

Copyright (C) 2019 American Society of Nuclear Cardiology.
}

studies, some of them randomized, have shown a higher incidence of lead concordance (LV lead located in a viable segment with the greatest delay in activation) when CRT was guided by echo imaging, which in turn resulted in better outcomes. ${ }^{6,7}$ One prospective study showed that the combination of the above two criteria in patients who have baseline LV dyssynchrony measured by SPECT resulted in improved LV synchrony after CRT which was associated with improved outcome. ${ }^{8}$ Zhang analyzed data on 79 patients who underwent CRT and had SPECT, echo, and NYHA class assessments at baseline and 6 months after CRT. SPECT was used to determine LV synchrony using phase analysis of regional thickening (time-activity) curves, an approach to dyssynchrony assessment that is now well established. ${ }^{9}$ The "optimal" LV lead position was defined as the latest contracting viable segment, based on regional phase values. Since the deployment of the LV lead is determined by coronary venous anatomy and thus not fully under the control of the electrophysiologist operator, hierarchical recommendations were made for two alternate lead positions: the " 2 nd recommendation" of a viable segment with a phase value within 10 degrees of the optimal segment and a "3rd recommendation" of viable segments which were adjacent to the optimal segment. Based on prior data indicating non-response, the apical and septal segments were excluded from consideration. Lead concordance was determined by identifying the lead position on a CT venogram performed within 7 days of CRT, and its association with the outcome measure of all-cause mortality, heart failure hospitalizations, and LV volumetric response $(>15 \%$ reduction in the LV end-systolic volume) was determined. Two prospective studies, Targeted Left ventricular Lead Placement to Guide Cardiac Resynchronization Therapy (TARGET) ${ }^{7}$ and Speckle Tracking Assisted Resynchronization Therapy for Electrode Region (STARTER) ${ }^{6}$ have previously 
Table 1. Association between left ventricular lead concordance and patient outcome

\begin{tabular}{|c|c|c|c|}
\hline Study & $\begin{array}{l}\text { Lead concordance } \\
\text { (guided vs standard) }\end{array}$ & $\begin{array}{l}\text { LV volumetric response } \\
\text { (guided vs standard) }\end{array}$ & $\begin{array}{l}\text { Composite outcome } \\
\text { (guided vs standard) }\end{array}$ \\
\hline $\begin{array}{l}\text { Current study } \\
\mathrm{n}=79, \mathrm{~F} / \mathrm{U} 4.08 \text { years }\end{array}$ & $59.1 \%$ vs $34.2 \%$ & $75.6 \%$ vs $51.9 \%$ & $22 \%$ vs $51.9 \%$ \\
\hline $\begin{array}{l}\text { TARGET }^{7} \\
\mathrm{~N}=220 \mathrm{~F} / \mathrm{U} 2 \text { years }\end{array}$ & $61 \%$ vs $47 \%$ & $70 \%$ vs $55 \%$ & $10 \%$ vs $15 \%$ \\
\hline $\begin{array}{l}\text { STARTER }^{6} \\
\mathrm{n}=165, \mathrm{~F} / \mathrm{U} 1.6 \text { years }\end{array}$ & $85 \%$ vs $66 \%$ & $54 \%$ vs $26 \%$ & $23 \%$ vs $43 \%$ \\
\hline
\end{tabular}

Lead concordance was defined somewhat variably but using a similar theme in the 3 studies as LV lead positioned in the latest contracting viable or adjacent segments. LV volumetric response was defined in all studies as a $>15 \%$ reduction in LV endsystolic volume. The composite outcome was defined in all studies as all-cause mortality and heart failure hospitalization. All differences listed in this table between the guided and standard approach were statistically different in all three studies

investigated the association between LV lead concordance and CRT outcome using echocardiography to define the optimal lead location (Table 1). Individually and jointly, these studies establish a strong association between an optimal LV lead position and CRT outcome.

So, how might the data from these studies be used to improve patient care in the field of CRT? I offer two observations as potentially important. First, a significant proportion of patients had a concordant lead position serendipitously, usually in the lateral wall, using a standard approach to the CRT implant. Second, the three studiesldiscussed did not use contemporary class IA criteria for patient inclusion, i.e., patients were not required to have $\mathrm{LBBB}$ or a $\mathrm{QRS}$ duration $>150 \mathrm{~ms}$. The fact that CRT response rates are much higher with class IA criteria may offer some mechanistic insight, based on the pathophysiology of LBBB. Patients with LBBB usually have the greatest activation delay in lateral wall (Figure 1, Malhotra et al., unpublished data). Serendipitously, they are thus amenable to resynchronization by pacing the septum and lateral walls with biventricular pacing, which is the only form of multi-site pacing that is currently available for clinical use. The association between the QRS width and CRT response is less clear, since there is not a good correlation between conventional imaging measures of dyssynchrony and QRS duration. ${ }^{10}$ Furthermore, there is strong evidence that patients with a narrow QRS complex are not benefitted (and may even be harmed) by CRT even if they have imaging evidence of LV dyssynchrony ${ }^{11}$

Thus, a synthesis of the composite knowledgebase on CRT mechanisms seems to indicate that when contemporary class IA criteria (symptomatic heart failure, $\mathrm{EF}<35 \%$, QRS duration $\geq 150 \mathrm{~ms}$, and $\mathrm{LBBB}$ ) are used to select patients, response rates are high. In this population, imaging LV dyssynchrony may not offer

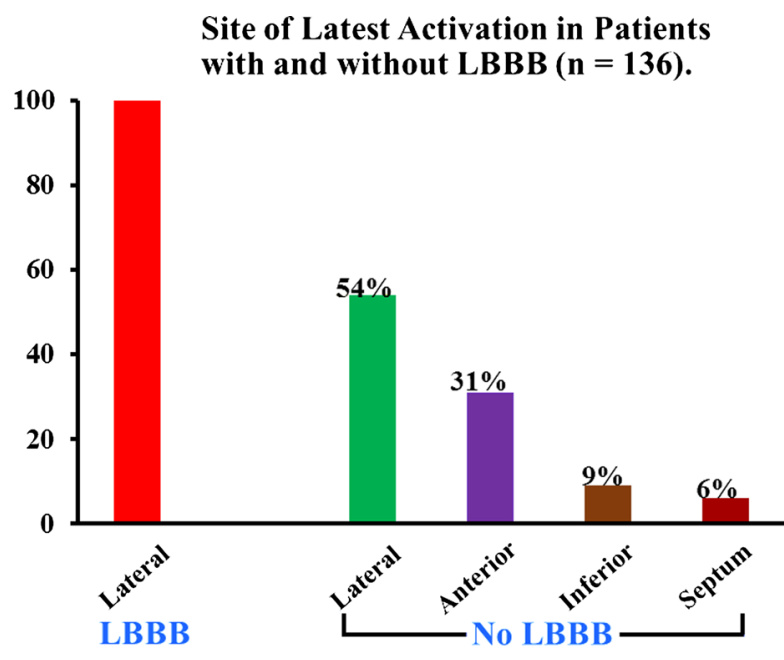

LV Wall with Latest Activation

Figure 1. Site of latest activated myocardial segment in patients with heart failure and LV ejection fraction $<35 \%$. LV regional dyssynchrony was determined using regional phase analysis of myocardial SPECT (Malhotra et al., data unpublished, presented in abstract form only).

added value (imaging for scar burden and location might still be important). However, for patients with QRS durations between 120 and $150 \mathrm{~ms}$, and for those with non-LBBB patterns of QRS morphology, imaging to identity the optimal position of the LV lead may be important to optimize response rates. Notably, in the TARGET study and the current study, latest activated segments outside the lateral wall were encountered, albeit less frequently, while these segments were excluded from analysis in STARTER. Whether this observation was more frequent in patients with nonLBBB QRS morphology was not analyzed. A future study to assess image-guided LV lead placement in 
patients with QRS durations between 120 and $150 \mathrm{~ms}$ and non-LBBB QRS morphology could be particularly illuminating.

\section{Disclosure}

Prem Soman and Saara Sillanmäki have no disclosures relevant to this editorial.

\section{References}

1. Tracy CM, Epstein AE, Darbar D, et al. 2012 ACCF/AHA/HRS focused update of the 2008 guidelines for device-based therapy of cardiac rhythm abnormalities: A report of the American College of Cardiology Foundation/American Heart Association Task Force on Practice Guidelines and the Heart Rhythm Society. [corrected]. Circulation 2012;126:1784-800.

2. Epstein AE, DiMarco JP, Ellenbogen KA, et al. ACC/AHA/HRS 2008 Guidelines for Device-Based Therapy of Cardiac Rhythm Abnormalities: A report of the American College of Cardiology/ American Heart Association Task Force on Practice Guidelines (Writing Committee to Revise the ACC/AHA/NASPE 2002 Guideline Update for Implantation of Cardiac Pacemakers and Antiarrhythmia Devices): Developed in collaboration with the American Association for Thoracic Surgery and Society of Thoracic Surgeons. Circulation 2008;117:e350-408.

3. Birnie DH, Ha A, Higginson L, et al. Impact of QRS morphology and duration on outcomes after cardiac resynchronization therapy: Results from the Resynchronization-Defibrillation for Ambulatory Heart Failure Trial (RAFT). Circ Heart Fail 2013;6:1190-8.
4. Blank. To be inserted later.

5. Adelstein EC, Tanaka H, Soman P, et al. Impact of scar burden by single-photon emission computed tomography myocardial perfusion imaging on patient outcomes following cardiac resynchronization therapy. Eur Heart J 2011;32:93-103.

6. Saba S, Marek J, Schwartzman D, et al. Echocardiography-guided left ventricular lead placement for cardiac resynchronization therapy: Results of the Speckle Tracking Assisted Resynchronization Therapy for Electrode Region trial. Circ Heart Fail 2013;6:427-34.

7. Khan FZ, Virdee MS, Palmer CR, et al. Targeted left ventricular lead placement to guide cardiac resynchronization therapy: The TARGET study: A randomized, controlled trial. J Am Coll Cardiol 2012;59:1509-18

8. Friehling M, Chen J, Saba S, et al. A prospective pilot study to evaluate the relationship between acute change in left ventricular synchrony after cardiac resynchronization therapy and patient outcome using a single-injection gated SPECT protocol. CircCardiovascImaging 2011;4:532-9.

9. Chen J, Garcia EV, Bax JJ, Iskandrian AE, Borges-Neto S, Soman P. SPECT myocardial perfusion imaging for the assessment of left ventricular mechanical dyssynchrony. J Nucl Cardiol 2011;18:685-94.

10. Bleeker GB, Schalij MJ, Molhoek SG, et al. Relationship between QRS duration and left ventricular dyssynchrony in patients with end-stage heart failure. J Cardiovasc Electrophysiol 2004;15:5449.

11. Ruschitzka F, Abraham WT, Singh JP, et al. Cardiac-resynchronization therapy in heart failure with a narrow QRS complex. N Engl J Med 2013;369:1395-405.

Publisher's Note Springer Nature remains neutral with regard to jurisdictional claims in published maps and institutional affiliations. 